\title{
Deconstructing Black physics identity: Linking individual and social constructs using the critical physics identity framework
}

\author{
Simone Hyater-Adams, ${ }^{1}$ Claudia Fracchiolla, ${ }^{2}$ Tamia Williams, ${ }^{3}$ \\ Noah Finkelstein, ${ }^{4}$ and Kathleen Hinko ${ }^{5}$ \\ ${ }^{1}$ ATLAS Institute, University of Colorado Boulder, Boulder, Colorado 80309, USA \\ ${ }^{2}$ University College Dublin, School of education, Belfield, Dublin 4, Ireland \\ ${ }^{3}$ Ohio State University, 191 West Woodruff Ave, Columbus, Ohio 43210, USA \\ ${ }^{4}$ Physics Department, University of Colorado Boulder, Boulder, Colorado 80309, USA \\ ${ }^{5}$ Department of Physics and Astronomy, Lyman Briggs College, Michigan State University, \\ East Lansing, Michigan 48824, USA
}

(Received 22 February 2019; published 9 August 2019)

\begin{abstract}
Understanding the ways in which Black folks identify as physicists can provide useful information about the facets of the physics discipline that perpetuate systems of oppression. In prior work, we developed the critical physics identity (CPI) framework to identify the structures and systems that impact the ways that people identify with the physics discipline. In this paper, we apply the CPI to deconstruct the factors that influence physics identity for a sample of undergraduate, graduate, and professional Black physicists. Using the CPI constructs, we classify important internal and external factors that influence how they identify, or not, as a physics person. We find that racialized resources were more influential than physics identity constructs, and document the subcodes of each CPI construct that are discussed most frequently among participants. We note variations between early and later career physicists and between men and women.
\end{abstract}

DOI: 10.1103/PhysRevPhysEducRes.15.020115

\section{INTRODUCTION}

Black students are underrepresented in the physics discipline, with bachelor's degrees declining in the last several years [1]. There is a growing body of work looking at what causes this lack of representation. Much of this work is situated in studies of identity, and how Black students negotiate the complex identities required of them as they pursue careers and studies in science, technology, engineering, and math (STEM) [2,3]. While these identity studies are necessary for the goal of understanding Black physics experiences, there is room for broadening the discussion to include perspectives that focus on systemic, institutional, and structural factors that influence identity development. With numerous successful initiatives (programs, pipelines, etc.) designed to recruit Black students to physics, many Black students still choose not to enter or to leave the discipline. This persistent trend indicates the need for a larger cultural change in order to support Black folks in the field. This work uses a framework that bridges "theory" and "practice," and simultaneously expands the discussion of identity in physics

Published by the American Physical Society under the terms of the Creative Commons Attribution 4.0 International license. Further distribution of this work must maintain attribution to the author(s) and the published article's title, journal citation, and DOI. education to consider how it is influenced, implicitly and explicitly, by systemic and institutional factors.

\section{BACKGROUND}

In order to foreground the impact of structures and environments on individual experiences, we look at the intersection of identity and culture. Research shows that physics culture includes barriers that Black students must persist through in order to remain in STEM. We inform the current study from the literature on experiences of Black students in STEM broadly [2,3], and the many identity studies about women of color in physics [4-7]. A feature of physics culture that is at the root of these barriers is a "culture of no culture" ideology, which is a pervasive belief that physics is objective and hence not biased [8]. This ideology makes it difficult to advocate for the people who experience marginalization in society and more specifically in the physics field. Reports on the experiences of underrepresented groups in physics give a wide array of examples of how physics culture excludes them in explicit and implicit ways [9]. Black students often report feelings of isolation and experiencing discrimination in their departments $[2,3,10]$. These are ways that structural racism and sexism impact Black students' mental health and wellness while pursuing degrees and careers in STEM fields [11]. McGee argues that research on Black students in STEM often does not examine 
the social and emotional stressors that impact the well being of these students [12]. In addition, students who persist and become faculty, they often end up doing extra "service" work, such as mentoring all of the students on color, while also being paid less than their white colleagues [13].

While there are a broad array of studies of race and identity in physics, we situate our work within STEM identity work and studies that report on Black experiences in physics. Research on the experiences of Black undergraduate physics students provides a useful framing of the struggles we continue to study here. In one study, students report that "race matters" in their experiences studying physics and dealing with physics faculty [14]. Students had different perceptions of how their race impacted their experiences, as well as their own responsibilities for finding resources to cope with them. Examples of racialized experiences that students described included pressures to represent their race, and feelings of being judged differently because of their race. In a similar study on Black women in physics, students report on the common themes of experience of a group of successful Black women physicists [15] having exposure to and academic preparation in the sciences, feeling supported by physics departments at HBCUs, coping with negative faculty interactions, and managing an academic and social life balance. Many of these themes are echoed in Rosa and Mensah's work studying the narratives of six Black women physicists [10]. Similarly, a study on intersections of race, gender, academic ability, and class for Black students in physics describes the importance for Black students to feel like they can be their whole selves while pursuing the major [16]. A common theme of experience for students of color in academia in general and STEM specifically is the feeling of existing in two worlds and having fragmented academic and social lives. This theme from Fries-Britt's work is used to stress the importance of intersectional studies that investigate how all of the identities a Black student might have in addition to their race shape their experiences.

Identity studies in physics education research often frame the physics community as a community of practice [17]. In the community of practice framework, Lave and Wenger establish that membership is accomplished through participation and engagement with the community of practice and it is this membership that is connected with our identity. Therefore, we situate this work in the idea that developing an identity as a member of the physics field is an important aspect of participation in the field, and that "physics identity" impacts the participation of women in physics [18]. Studies of identity at the intersection of physics, race, and gender provide insight into the struggles that students of color experience, and the strategies they employ to continue in the discipline. Much of the work on physics identity stems from work focused on the experiences of women of color, broadly in STEM and in physics specifically [4-7,19]. These studies use different frameworks of identity to understand the ways that women of color navigate their intersecting identities as they progress through their STEM careers. The framework for physics identity that we draw from has roots in a science identity framework that was applied to analyze the experiences of women of color who were students in various STEM departments [4].

\section{THE CRITICAL PHYSICS IDENTITY FRAMEWORK}

In this work, we use the critical physics identity framework (CPI) [20], an approach we developed in prior work that broadens the focus of physics identity to includes the individual's sense of self and the impacts of structures around them. This framework for identity was developed for use in analyzing how institutional and systemic factors impact the development of physics identity. In doing so, we draw from sociocultural perspectives of identity and we use a lens that stresses the importance of considering one's environment and its impact on identity development [21]. The CPI framework employs constructs from Hazari et al.'s physics identity framework [18] that focuses on the individual and couples with Nasir's racialized identity resources framework [22] in order to consider the impacts of structural resources that can impact one's physics identity. The physics identity constructs include recognition, interest, competence/performance. The racialized identity framework has three broad resource constructs that can connect a person to a practice: ideational, material, and relational. Figure 1 shows the model for the CPI which modifies these frameworks into one that includes six constructs: recognition, interest, competence/performance, relational resources, material resources, ideational resources. The CPI redefines

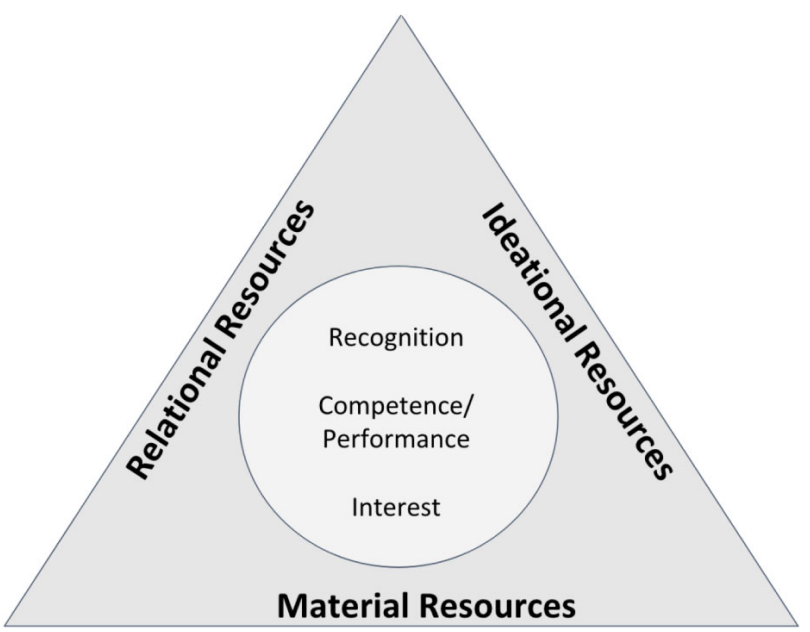

FIG. 1. An updated working model for the critical physics identity framework [20] that depicts how we see the structure of the six constructs based on how they interact. It includes constructs for physics identity (recognition, interest, and competence/performance) and racialized identity (relational resources, material resources, and ideational resources). 
recognition as being recognized (or not) as a physicist or physics person, interest as having an interest in the physics field, competence/performance as the belief in one's ability to understand the content and perform required tasks. It also redefines relational resources as aspects of relationships that impact participation in physics, ideational resources as aspects of an idea that impact participation in physics, respectively, and material resources as physical things that can lead to other constructs.

In order to operationalize these constructs to document the experiences of Black physicists through coded interviews, specific subcodes for the CPI constructs were developed. Two of these subcodes that we use to categorize several CPI constructs are positive or negative and internal or external. We define positive and negative subcodes as encouraging or discouraging participation in physics. We define internal subcodes as a person talking about their own ideas and external as a person talking about other people's ideas. In addition, ideational resources have four unique subcode categories: positioning in physics, what is valued in physics, perceptions of physicists, and personal characteristics. The findings in this paper inform some small additions to the CPI interest construct. This includes the addition of a new subcode called teaching and outreach that we code for when participants talk about their interests in physics teaching and outreach. We include the CPI code structure and full definitions in Table II of Appendix A. When using the CPI constructs to analyze stories, we see that the constructs relate to one another, and understanding the patterns by which these constructs show up and interact within one's story brings out useful knowledge about the identity they have developed as a member of the physics field. Together, the CPI framework provides information that paints a more holistic picture of the complex ways in which Black people can identify as physicists, as well as how their unique experiences in the field mold these complexities. This informs potential key levers for creating positive educational experiences for Black folks in physics.

To begin to identify the patterns of interaction between institutional structures and individual experiences, this study seeks to document the unique identities of Black physicists and the experiences and resources that molded them. While our prior work focused on presenting this framework and methodology [20], this work explores how to use the CPI framework in order to capture the nuanced ways in which systems and structures can differently impact Black physicists' experiences in the field. We apply the CPI framework to a set of 11 interviews from Black physicists to document and analyze their experiences and begin to answer the following research questions: (i) What CPI constructs are important for supporting Black physics identity? (ii) What are ways in which connections between CPI constructs provide support for Black physics identity? From the analysis, we present findings that contribute to conversations in the literature seeking to understand structural, systemic, and institutional factors that impact the identities of Black physicists. Our findings highlight the importance of ideational resources, positive material resources, and recognition to our participants, as well as the significance we see in the ways that these constructs connected with each other and the other CPI constructs.

\section{POSITIONALITY STATEMENT}

As the first author, a driving force behind this work is my personal experience and struggles participating in and identifying with the physics community. Understanding my own identity as a physicist has been a journey that was strongly impacted by the racialized experiences I have to navigate on a daily basis. The CPI framework was developed with a particular intention for use in exploring racialized physics experiences like my own. These experiences, many of which echo the narratives of participants in these studies, equip me with a unique perspective that informs the analysis of this work. This paper presents findings that point to the importance of the ideologies that connect a person to physics, and how material and ideational resources can impact these ideologies.

My own story about applying to graduate school simultaneously shows how these CPI resources influenced the construction of my own physics identity. It also serves as a form of validation for the CPI framework as a tool to analyze racialized physics experiences. I decided I wanted to go to graduate school during the summer before my senior year of undergraduate studies. My undergraduate physics experiences were full of positive recognition from material resources like participation in research, receiving scholarships, as well as positive competence/performance from my ability to maintain above average grades. However, I learned about the required physics GRE test only a week before the only date available to take the test prior to application deadlines. Because of this limited study time, I did not do well on the test. However, because I had several research experiences, I held ideational resources that my positioning in the field was good enough that I might still have a shot at graduate school despite the poor score, which functioned as a negative material resource in my case. Because of this challenge, I leveraged a positive material resource, a nasa.gov email address, in order to seek out relational resources with professors in physics graduate programs that interested me. I put in the energy and time to contact professors in these departments, asking them about the possibilities of me being accepted in their program with my application package. Despite this extra effort, fueled by my positive internal recognition as a person who belonged in a physics program, I ended up getting rejected from all but one of the physics departments I applied to, specifically because of the GRE score. This rejection caused a shift in my ideational resources about what was valued in these programs. I was under the impression that research was the most important part of my application for graduate school, and 
having been involved in research for all four years of my undergraduate career, I was shocked at how heavily the GRE weighted into decisions about my capabilities. These material resources discouraged me from attending a traditional physics program for my graduate studies. Through the negative recognition from these material resources, the ideational resources I had about my positioning in the field shifted so that I believed a traditional physics graduate program was not for me. This serves as a useful example of how the resources along my path have impacted the ways that I participate in the physics discipline, as well as how I identify, and often not, as a physicist.

\section{METHODS}

\section{A. Data collection}

In this work, we draw from the methodology used to develop the critical physics identity framework [20]. The primary data sources for this study are semistructured interviews, and participants were recruited through referrals from individual networking by members of the research team as well as advertising through social media. Interviews were conducted by the two Black women (authors 1 and 3) in the research group and lasted 49 minutes on average (see Appendix B). The other members of the research team included a Latina woman, a white woman, and a white man. Interviews were semistructured and used a protocol of questions that prompt participants to tell personal narratives, or stories about their experiences pursuing physics as a career, as well as their childhood experiences with physics and science broadly. We use a lens of narrative inquiry [23], which is grounded in the idea that there is value in what a person decides to include in their stories. Therefore, this protocol captures the important events and lines of thinking that mold the stories our participants tell. The protocol included questions such as "How did you end up in physics?" and "How has participating in physics impacted your life?" After each interview, we asked participants to fill out a demographic survey. Interviews were mostly conducted via a video conferencing service and the recorded audio was transcribed by a third-party consultant. In our previous work, we operationalized the CPI constructs through an iterative process and validated the constructs and subcodes with our research team [20].

The participants in the sample used for this work includes 11 physicists who identify as Black, African, and African American who range from upper-level undergraduates to professional physicists practicing research and education. We define "physicists" as anyone who is close to receiving (within a year) or already has received a bachelor's degree in physics. Because we see the inability to cultivate a healthy physics identity as something that contributes to the trend of Black folks not choosing to enter, and deciding to leave the physics discipline, we look to Black physicists who have remained in the physics discipline. We do this to understand the factors that contribute to the development and maintenance of their physics identities, and therefore interview physicists at all levels of participation, from entry level (e.g., undergraduate) to more senior positions. In the sample, there are three undergraduate students, four graduate students, and four professionals, which we define as people who hold post-doc, faculty, and staff positions or are working in jobs outside of the academy. Roughly half of our sample identify as women and half identify as men. We also note that there are members of our sample who indicated they identify as LGBTQ as well as members of our sample who are international students. Because of the nature of our small sample size, and the small numbers of Black physicists in the field, concerns of anonymity make us unable to report on more detailed information about our participants.

\section{B. Analysis methods}

The data collected were analyzed using both quantitative and qualitative methods. We used qualitative analysis software in order to code the transcripts of each interview subject using the CPI constructs presented in Appendix A. We note here that all of these constructs are coded when the participants talked about them in connection with their physics experiences. For example, not all relationships that were mentioned were coded, only the ones that they described as impacting their participation in physics. The units for coding each construct were agreed upon amongst the broader research team and are based on one contained "idea" that is talked about by a participant for each construct. Transcripts were coded using an iterative validation method. First, the two researchers (first author and third author) who conducted the interviews coded each interview transcript separately. We ran an intercoder analysis of agreement between two coders on the transcripts at this point, and transcripts had a kappa value greater than 0.7 . Then, these two versions of the coded transcripts were reviewed by the same researchers together. This review consisted of discussions about the places in each interview where sections of the transcript were coded differently. When these disagreements could not be resolved amongst the two, they were flagged and brought to the larger group for further discussion. From the larger group discussion, which included the second and fifth authors, we developed more refined understandings of the CPI construct and subcode definitions. After these discussions, the two coders revisited the transcripts and recoded these spots and integrated the changes into one version of the transcript. For more detail on the coding scheme see the discussion of it in our prior work [20].

When the coded transcripts were finalized, we used the software to conduct qualitative and quantitative analyses. There are three ways we parse the data for analysis in order to answer our research questions: (i) we aggregate counts of CPI constructs and subcodes across all subjects, (ii) we find the points of overlap between coded CPI constructs, and 
(iii) we examine the variation in specific CPI codes for each of the individuals interviewed. Our first level of quantitative analysis focuses on the code counts or the number of times a construct is coded across our entire sample. For each interview subject, all CPI codes and subcodes are counted. We also documented overlaps between constructs, by using the MAXQDA software to identify the instances in the transcripts where constructs were coded together. Tables of the individual CPI code counts and the overlapping codes are created for each interview subject, across interview subjects, as well as binned by gender and level of education.

These counts, in addition to providing the quantitative findings presented below, help us identify the most prominent constructs that are examined in more detail through a qualitative approach in order to understand the themes discussed by our participants. To look at themes within each interview in more depth, we create summaries for each participant's transcript that aggregate the coded sections of transcript by each CPI construct and subcode. These summaries are then compiled into one large document that aggregates the summarized transcripts from every participant. Each profile is then analyzed in two fashions: (i) Identifying themes across CPI codes by examining the areas of transcripts where constructs are frequently coded together, and (ii) identifying themes within the CPI codes by summarizing the main themes of the coded segments under each construct. To identify connections across constructs, we use the MaxQDA Code Relations browser, which counts each time two codes are coded within the same segment of transcript in the sample of interviews. Using the Code Relations Browser, we are able to identify which constructs our participants talk about together frequently in the interviews. From these themes, we are able to draw insights into the factors that have molded the ways these Black physicists identify with the physics discipline.

Interview validation.-While this study draws from a small sample size $(N=11)$, which can be seen as a potential limitation, our analysis and findings draw heavily from our qualitative data. We also note that the statistical tests used in our analysis are nonparametric to support small sample size, and are exclusively used to show significant differences in our code counts. The interview questions we asked were designed to elicit a rich and nuanced picture of people's experiences, which, in this data set, is not dependent on the duration of the interview. For example, Andrew, in $40 \mathrm{~min}$ discussed 88 elements that were coded, while Erica, in 64 min has 67 codes. Thus, the duration of the interview does not determine the depth or richness of the data. Even with a small data set, we find the Pearson correlation between code counts and interview length negligible $(r=0.0679$, $p=0.0108$ ). In Table III of Appendix B we show a table of the duration of each interview, the number of codes in each interview, as well as the ratio of code count per minute. These data suggest that the number of codes per interview was not significantly impacted by people who might have talked at greater length. As such, we believe that our interviews are not missing significant components of people's stories due to the interview duration.

\section{FINDINGS}

Our analysis of this sample of Black physicists using the CPI framework provides findings that not only validate the ability for the CPI to highlight important factors beyond what has been captured by previous frameworks [18], but also gives us some insights into the patterns of experience amongst the physicists within the sample. This section outlines the findings from the two forms of analysis: examining the code counts for the CPI constructs across our sample of transcripts and examining the codes that overlap often in the coded transcripts. From the distribution of all the codes in each interview, we focus in on the constructs that were coded most for all participants and further examine those areas of the narratives in the transcripts. Similarly, we also find the codes that overlap most in the interviews and examine those areas of the narratives in the transcripts. From this process, we find that ideational resources, recognition, and material resources are the most prominent CPI constructs for our sample.

\section{OVERALL ROLE OF CPI CONSTRUCTS}

Figure 2 depicts the total code counts per construct for each participant in our sample. These counts are normalized to the total code counts for each individual interview. All six CPI constructs show up for everyone with variations of how often they show up for each participant, which further suggests to us that CPI is providing us with useful and unique information across our sample. Additionally, the constructs show up in different orders within the transcript for each subject, also demonstrating that variations are due to capturing participant experience instead of an artifact of the interview protocol. When looking further into these variations, we see that the racialized identity resource constructs (material, ideational, and relational resources) together make up a larger percentage of the total codes for each participant than the physics identity constructs (recognition, interest, competence/ performance). In order to test the variations within the CPI constructs, a dependent sample's nonparametric Wilcoxon signed-rank test was conducted [24]. This test was found to be statistically significant, $V(1)=66, p<$ $0.005 ; d=2.56$. The effect size for this analysis $(d=2.56)$ was found to exceed Cohen's [25] convention for a large effect $(d=0.80)$ [25]. These results indicate that the subjects in this sample draw consistently more from their racialized resources $(69.8 \%)$ than their identity resources $(30.2 \%)$. In particular, we note the resource constructs (drawing from racialized identity) are key factors to the experiences of our participants in their construction of 


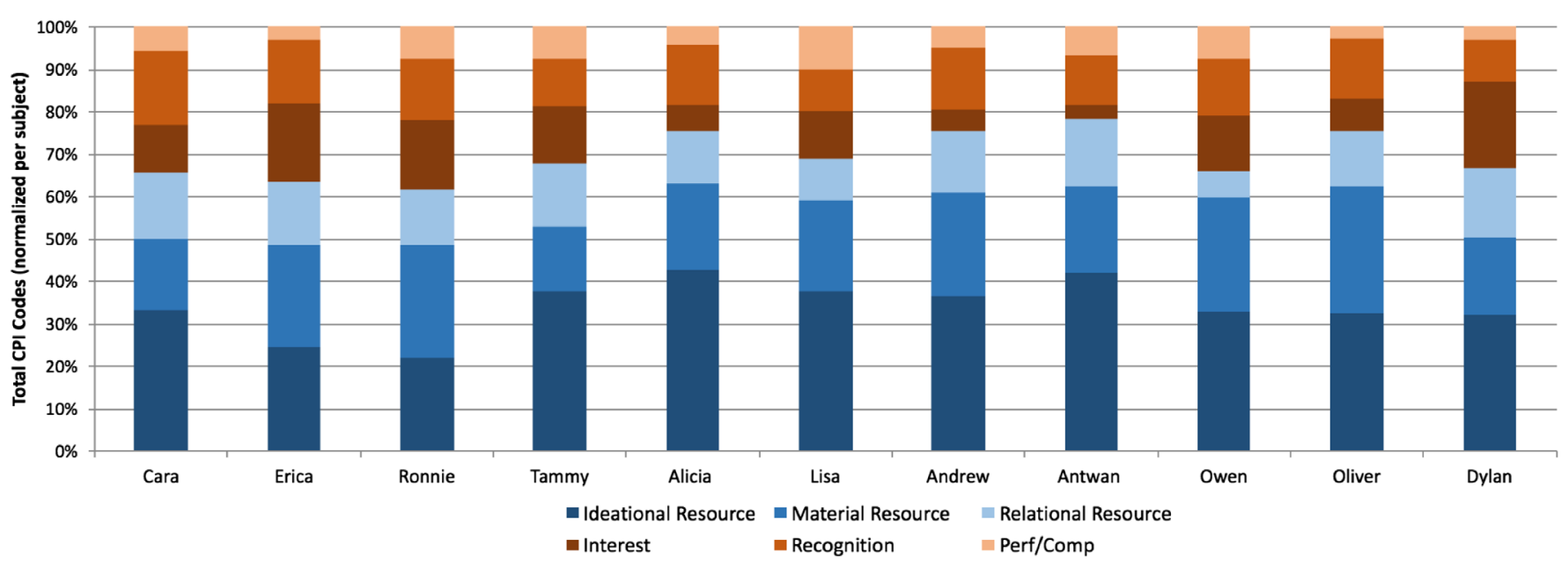

FIG. 2. CPI constructs for each participant normalized to the total code counts for each individual interview. Constructs come in two types, physics identity (orange shades) and racialized resources (blue shades). The graph shows the large difference between the percentage of physics identity constructs and the racialized identity resource constructs coded for this sample.

physics identity. Additionally, we see an overlap of the physics identity and racialized identity constructs, further demonstrating the utility of the CPI. These data show us that combining and building on the two separate frameworks of physics identity [18] and racialized identity [22] in the CPI, we are gathering more information about individuals' identities than is captured by these prior frameworks separately.

Overall, Fig. 2 provides insight into the role that the racialized resources play in the construction of physics identity and the resources that our sample discussed the most in their narratives about their experiences pursuing the physics field. We see that for our participants, ideational resources was the most coded construct, averaging $34.2 \%$ of the constructs coded in each interview. We also see that material resources were the second most frequently coded construct with these codes, averaging $22.2 \%$ of the constructs coded in each interview. When looking at the other CPI constructs, relational resources and recognition both make up an average of $13.3 \%$ of the codes in each interview, while interest codes make up $11.7 \%$ and competence/performance make up only $5.2 \%$. The low numbers of competence/ performance codes suggest to us, for this sample, that one's belief in their ability to understand the content and perform the required tasks might not influence their physics identity as much as the other factors included in the CPI. In the following sections, we outline the themes and trends we find from our analysis of this sample using the CPI. We present the themes found from the code counts of the most coded physics identity construct and racialized identity construct (ideational resources and recognition), as well as the codes that connected most (material resources, internal positioning ideational resources, and recognition).

\section{A. Ideational resources}

Because ideational resources represent $34.2 \%$ of our sample, we take a deeper look into the themes brought out by the subcodes. The ideational resources construct of the CPI has four subcodes: (i) how they themselves and others view their positioning in physics, (ii) what they see as valued in the physics field versus what others see as valued, (iii) personal characteristics that they and others see as valuable to the physics discipline, and lastly, (iv) the perceptions they have and other people have of physicists. Figure 3 depicts the four subcodes of ideational resources for each participant: positioning and physics, perceptions of physicists, what is valued in physics, and personal characteristics. Each subcode is also coded as internal or external, which denotes the source of the ideational subcode-internal (from the self) or external (from others).

A clear outcome of this analysis is the dominance of internal versus external ideational codes. Participants in this study were much more likely to discuss ideologies that they hold themselves than to talk about the ideologies of others. Across all the interviews in our sample, the average percentage of internal ideational constructs coded was much more frequent than external ideational constructs. We use the Wilcoxon signed-rank test [24] again to test the significance of this difference. This test was found to be statistically significant, $V(1)=65, p<0.005$; $d=$ 3.30. The effect size for this analysis $(d=3.30)$ exceeds Cohen's convention for a large effect $(d=0.80)$ [25]. These results indicate that the subjects in this sample draw consistently more from internal ideational codes $(25.33 \%)$ than external ideational codes $(7.74 \%)$. Of all the ideational resources discussed by participants, internal positioning in physics was the subcode talked about most, whereas external positioning was among the least discussed resource. When the physicists in our sample talk about their positioning in physics, they all discuss the broad idea of feeling like they both do and do not feel like they are a physicist. This framing holds even for the three participants, Cara, Erica, and Antwan, who do not have a large percentage of positioning codes. 


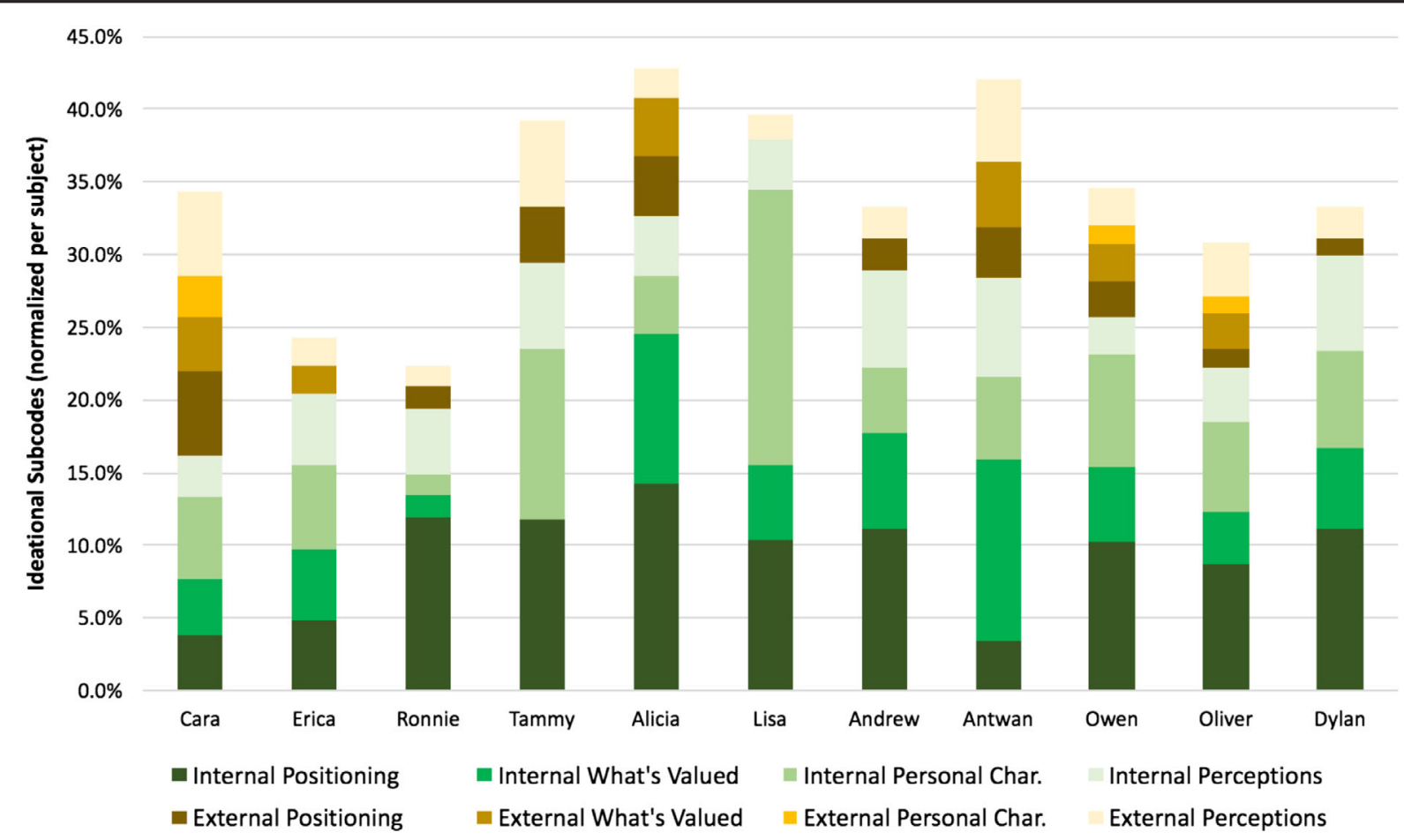

FIG. 3. Graph of the internal and external ideational codes for each participant, normalized to the total code counts for each individual interview. Shades of green represent internal subcodes and shades of brown represent external subcodes.

A common theme that participants discussed was the significance of their Black identities as it relates to their position in physics. For example, Ronnie listed the identities they felt were most important to them as "Black, female, and then physicist." Participants also talk about feeling like they do and do not belong in the field. Tammy discusses not feeling she belongs in certain physics spaces when she states "Sometimes yes, sometimes no. (laughs) Sometimes yes. I mean it depends on the crowd. Sometimes you go somewhere and you don't feel like you quite belong." In addition, many participants hold the idea that traditional physics is not what they want to do for their career. Oliver discusses this perspective when he states "Like a part of the reason why I don't say I'm a physicist is because that's not my ambition for life." Similarly, Lisa sees her pursuit of a physics bachelor's degree as a "means to an end."

From the internal resources shown in Fig. 3, we see that many participants also discuss what they see as valued in physics as well as some of their personal characteristics. Most participants talk about the ways that abstract concepts, rigorous research, and status as a professor are all characteristics of physics that are valued in the field. They also discuss the ways that they value other aspects of the field, such as teaching, mentoring, and feeling it is their duty to provide representation for Black physicists to come. The values they discussed are also often described as reasons why they are still pursuing the field. When asked what motivated him to continue, Andrew responded:
"Honestly it was me looking at the situation and saying there's not that many Black people in physics, I have to be one of these people". Many of the personal characteristics our participants described included traditional physicist characteristics, like being a logical and analytical thinker and a hard worker. However, many participants also mention the mental and emotional challenges that they have experienced while pursuing the field. This theme was salient in Lisa's interview, who's personal characteristic ideational resources focused mostly on this topic: "So physics really challenged me mentally and emotionally because I was like whoa, this stuff is hard, and I can't be mediocre as I'm doing, I have to put in the effort and I have to at least try to understand this."

The external codes made up a small percentage of the ideational resources discussed by members of our sample. Only three participants brought up external personal characteristics in their interviews and this code on average made up less than $1 \%$ of all codes in these interviews. The most frequently coded external construct, which on average made up $3.2 \%$ of each interview in the sample, was perceptions of physicists, followed by positioning with a $2.3 \%$ average, and what is valued in physics with a $1.8 \%$ average. When participants discussed external perceptions of physicists, they were often talking about the ways that people inside and outside of the discipline view the stereotypical image of physicists, and how this leads to people making assumptions and judgments about them. We see this in an example from Oliver's interview when he talks about social interactions 
with people outside of physics when they learn he is a physicist "Because I guess a lot of the distance that gets created is oh you're in a science that a lot of people aren't in, and when they are there's like a stereotype that there aren't very good social skills associated with a physicist."

We find it interesting that the external subcodes for positioning and what is valued in physics showed up less in interviews. When participants talked about external ideas about what is valued in physics, they talked often about the pressure not to engage in outreach or any nonresearch related activities. Antwan discussed this in his interview: "At the same time I was also taught that too much outreach is bad. A lot of times people want to make sure that they see you in the lab, they want to make sure that you are working, so if you spend more than $10 \%$ of your time doing outreach you're doing something wrong. So I can't do too much, I can't do as much as I'd like to do." When external positioning comes up in the interviews, participants are talking about how others do or do not see them as a physicist. In response to a question asking whether others see her as a physicist, Tammy says "So it depends what forum I am in, like and depends on the crowd basically you're hanging around with. At first glance, no, but when you start talking about things yes, then people can identify that you're a physicist. But it's one of those things that, it's very hard for people to identify that you're a physicist or something like that." While many of these external ideational resources were coded less frequently than the internal resources, the themes that come from external resources are still telling. In addition, a few of the external positioning and what is valued codes overlap with recognition, which gives another indication that recognition can be an important factor to interrogate in this sample. We do this in the next section, highlighting the internal and external findings from the recognition codes in our sample.

\section{B. Recognition}

As discussed in the background section, we know from the literature that recognition has been highlighted as an important factor in the development of a physics identity. We see a similar indication of this importance in the findings from our participants. Of the physics identity constructs in the CPI, recognition was most prominent across the sample, making up an average of $13.3 \%$ of all the codes in each interview compared to the $11 \%$ average of interest codes and $5.8 \%$ average of the competence/performance codes. Figure 4 shows the fractions of recognition codes that arise from the self (internal) or others (external) for each participant, and participants are organized by position in their physics careers. When discussing external recognition almost every person in the sample discusses having family and friends, mostly outside of the discipline, who see them as a physicist. The trend in the graph shows that the amount of external recognition discussed by participants is higher for folks who hold more advanced positions in the field, while internal recognition decreases.

The average percentage of internal recognition is $6.27 \%$ for professionals, $5.48 \%$ for graduate students, and $8.28 \%$ for undergrads in our sample, while the average percentage of external recognition is $9.08 \%$ for professionals, $6.10 \%$ for graduate students, and $2.98 \%$ for undergrads. Thematic analysis of these codes also confirms this trend. Participants at the more entry-level positions report feeling like they do not completely belong in physics and do not completely identify as a physicist. This trend is shown in the difference

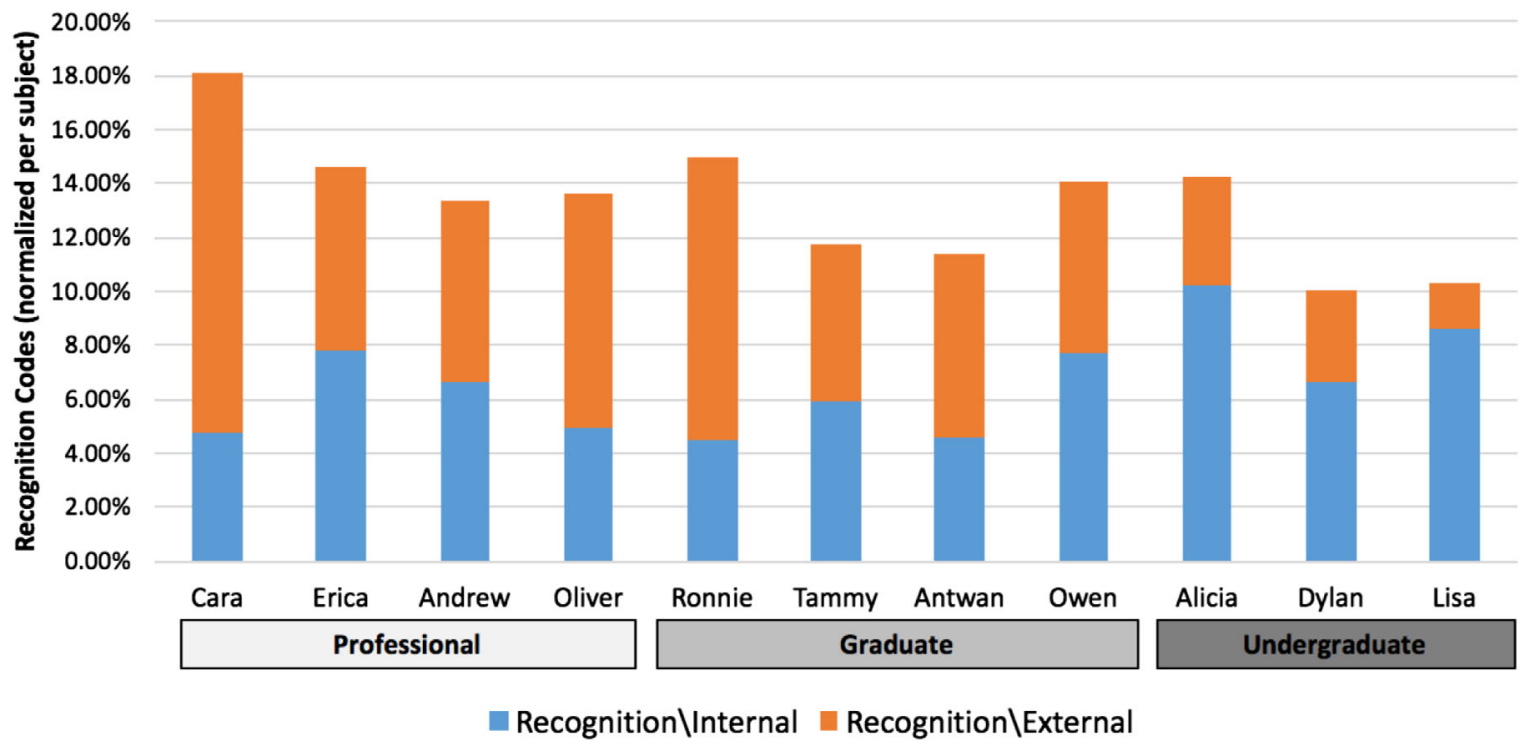

FIG. 4. Breakdown of internal and external recognition codes for participants based on their career position. The counts are normalized to the total code counts for each individual interview. The blue parts of the bar represent internal recognition percentages, and orange parts of the bar represent the external recognition percentages. 


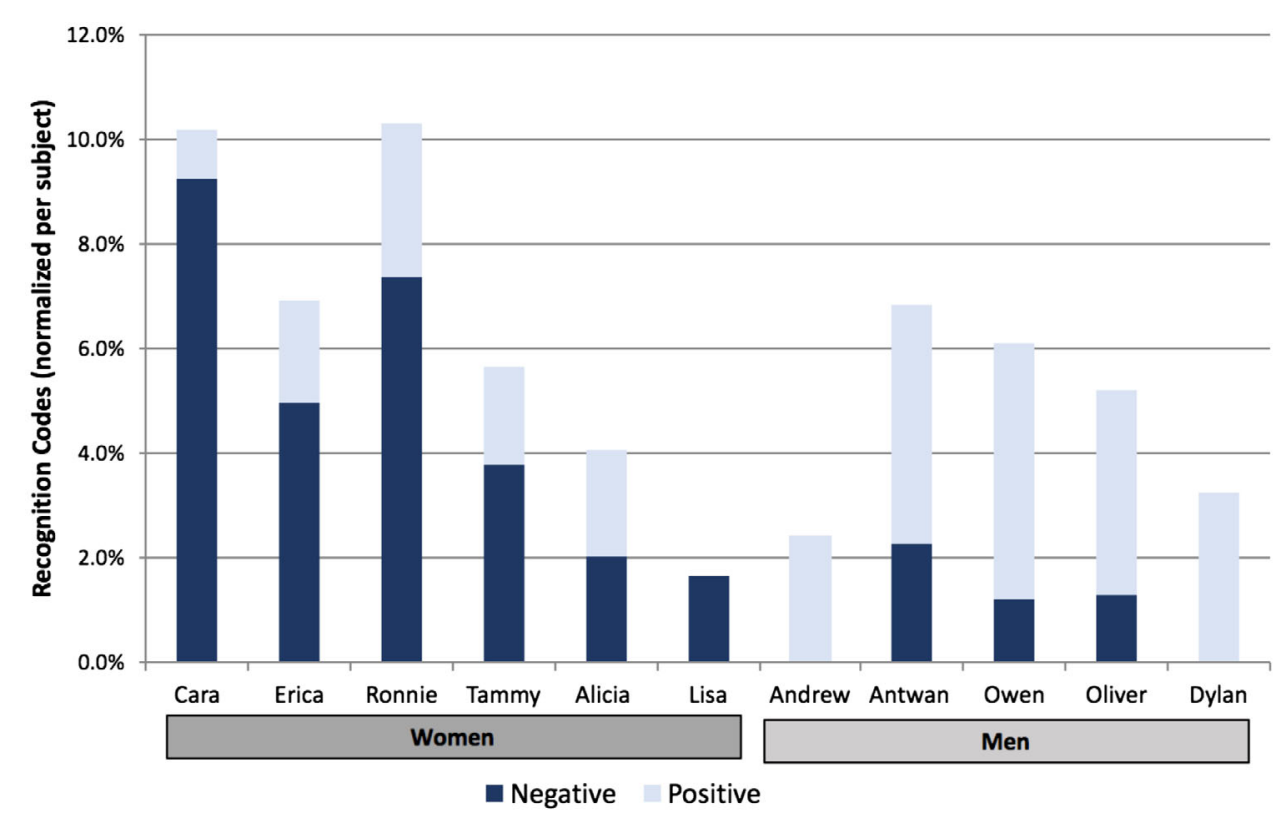

FIG. 5. Breakdown of external recognition for men (to the right) and women (to the left) in our sample, normalized to the total code counts for each individual interview. The dark blue bar represents the percentage of negative external recognition, and the light blue represents the percentage of positive recognition.

between positive and negative recognition. For example, Dylan who had recently received his bachelor's degree in physics when interviewed, states "Definitely I have a curiosity about the universe, how it works, how things relate to each other. So, based on those terms, yeah. I study physics, so I would say I'm a physics person, yeah." He continues on to clarify his response, mentioning his position in the field as a factor: "but I almost hesitated calling myself a physicist just because I haven't had a higher level of training and studying physics." The internal recognition discussed by the participants at more senior levels was largely positive. The professionals in the sample all explicitly state that they identify as a physicist or physics education research, and discuss struggles coming to these identities. Erica, a professor of physics, asserts her identity as a physicist when stating "I would say probably that's my strongest identity, is as a physicist. Which is why I want people to see me as a physicist, because that's how I most strongly identify." but later addresses her struggles to feel like she belongs in the community when stating "I mean yes and no. I mean I always felt like physics was what I'm supposed to be doing, or at least the venue through which I'm supposed to teach. But you know, do I feel a fully included member of the physics community? Probably not."

We see a clear difference in the predominant types of recognition that men and women discuss in the sample. Figure 5 shows the percentages of positive and negative external recognition of each participant but aggregated for men and women. The external recognition discussed by men in our sample is predominantly positive recognition, meaning it encourages their participation in physics. They talk about feeling like their colleagues, peers, and superiors see them as a physicist as well as receiving praise from their families for pursuing the discipline. Antwan talks about how his family looks up to him and how it gives him the ability to shift their perspective: "So family life has been impacted in a good way, but at the same time I'm able to show my family that there isn't a difference between a physicist and a teacher who might teach English." Conversely, the external recognition discussed by women in our sample is predominantly negative. Common experiences with negative external recognition for the women in our sample included experiences with having to prove their skills to peers and people senior to them, people being surprised to hear they studied physics, people not being confident in their success, and being ostracized from family and friends because they are seen as "too smart." An example of this is when Erica discusses how her family treats her differently: "[With] extended family, again, because most of them are sort of blue collar workers, they don't know- they don't know how to talk to me like I'm a normal person, even though I do talk about normal things." However, when these women discuss positive external recognition, they also describe the relationships with family and friends as the spaces where they are most seen as a physicist.

\section{CODE CONNECTIONS}

Because of the design of the CPI framework, we are able to examine when constructs "connect" to one another, which we define as two constructs that are often linked within a narrative. We see this connection in our interviews when constructs "co-occur," or are coded together often. In Table I we list the constructs and subcodes that co-occurred in our 
TABLE I. List of code connections from our sample that co-occurred 5 or more times across the 11 interviews. Each connection includes the names of subcodes that were coded together, the number of times they were coded together, and an example excerpt from an interview where this code connection occurs. The table is organized with the most coded constructs listed first and color-coded by construct (positive constructs have a lighter shade, and negative with bolder shade). We note that this organization does not imply directionality of the connection.

\begin{tabular}{|c|c|c|c|}
\hline Code connections & & Frequency & Example excerpt \\
\hline $\begin{array}{l}\text { Positive Material } \\
\text { Resource }\end{array}$ & Positive Relational Resource & 10 & $\begin{array}{l}\text { you had to take intro physics } 1 \text { and } 2 \text {, and when I took } \\
\text { those classes I also developed like really close } \\
\text { relationships with my professor. He was very } \\
\text { supportive. -Alicia }\end{array}$ \\
\hline $\begin{array}{l}\text { Positive Material } \\
\text { Resource }\end{array}$ & Positive Content Interest & 10 & $\begin{array}{c}\text { But what attracted me to the physics program was the } \\
\text { background that you get from what comes with the } \\
\text { physics curriculum at the school that I went to. -Oliver }\end{array}$ \\
\hline $\begin{array}{l}\text { Positive Material } \\
\text { Resource }\end{array}$ & $\begin{array}{l}\text { Positive Teaching and Outreach } \\
\text { Interest }\end{array}$ & 7 & $\begin{array}{l}\text { my sophomore year doing an REU at the University of } \\
\text { Washington in physics education research, and that's } \\
\text { where my interest in teaching was piqued. -Erica }\end{array}$ \\
\hline $\begin{array}{l}\text { Positive Material } \\
\text { Resource }\end{array}$ & $\begin{array}{l}\text { Positive Performance/ } \\
\text { Competence }\end{array}$ & 6 & $\begin{array}{l}\text { So I did physics in my high school and I ended up getting } \\
\text { an A. -Tammy }\end{array}$ \\
\hline $\begin{array}{l}\text { Positive Material } \\
\text { Resource }\end{array}$ & $\begin{array}{l}\text { Internal Positioning Ideational } \\
\text { Resource }\end{array}$ & 5 & $\begin{array}{l}\text { So I would say for the major part, probably if I didn't do } \\
\text { any outreach I don't think I would be in physics. } \\
\text {-Owen }\end{array}$ \\
\hline $\begin{array}{l}\text { Internal Positioning } \\
\text { Ideational } \\
\text { Resource }\end{array}$ & Positive Internal Recognition & 10 & $\begin{array}{l}\text { I definitely identified as a Black physicist because I've } \\
\text { got a bachelor's degree in it, like I earned the stripe. } \\
\text {-Ronnie }\end{array}$ \\
\hline $\begin{array}{l}\text { Internal Positioning } \\
\text { Ideational } \\
\text { Resource }\end{array}$ & Negative Internal Recognition & 8 & $\begin{array}{l}\text { Yeah, like I guess when I think about stereotypical } \\
\text { physicists I don't think I fit that-like, I don't think } \\
\text { I fit that persona. -Lisa }\end{array}$ \\
\hline $\begin{array}{l}\text { Internal Positioning } \\
\text { Ideational } \\
\text { Resource }\end{array}$ & Negative External Recognition & 5 & $\begin{array}{l}\text { So for some people the fact that my Ph.D. is in education } \\
\text { means to them I'm not a physicist. -Cara }\end{array}$ \\
\hline $\begin{array}{l}\text { Negative Relational } \\
\text { Resource }\end{array}$ & Negative External Recognition & 5 & $\begin{array}{l}\text { so in family life, I would say it's ostracizing. Not for my } \\
\text { immediate family so much, although a little bit with my } \\
\text { older brother. -Erica }\end{array}$ \\
\hline
\end{tabular}

sample 5 times or more. We note that positive material resources and internal ideational resources were the most connected constructs, with relational resources and recognition coming up less frequently amongst the most commonly connected codes. In this section, we report on these code connections that showed up most in our sample and give examples from the interviews about how these connections appear in participants' narratives of experience. While we acknowledge that less common connections might also provide us with useful insights, we narrow our focus to the most common for the scope of this paper.

\section{A. Positive material resources}

In our sample, positive (encouraging the participant to pursue physics) material resources were coded with at least one subcode from every other CPI construct. Because we define material resources as "material things that can provide access to other identity constructs," it is no surprise that this construct is often coded with other constructs. We find that the most common material resource subcode in our sample is positive, which was coded in all the interviews and made up an average of $12.58 \%$ of codes in each interview. While negative material resources were also brought up by each participant, they were discussed half as much, making up an average of $6.23 \%$ of codes in each interview. Negative material resources also connected much less with the other CPI constructs compared to the positive material resources. The strongest connection negative material resources had with another construct was 4 co-occurrences with internal positioning ideational resources. While positive material resources connected most with positive subcodes from other CPI constructs. The first five code connections listed in Table I are connections between positive material resources and other CPI constructs. These subcodes include positive relational resources, positive content interest, positive teaching and outreach interest, positive competence/performance, and internal positioning ideational resources.

One of the subcodes with the highest number of code connections, with 10 co-occurrences to positive material resources, is positive relational resources. In prior work, we discuss the importance of relational resources and the ways they connect to recognition [26]. In our current sample, positive relational resources made up an average of $9.3 \%$ of 
each interview, compared to the $2.83 \%$ average that negative resources made up. When connected to positive material resources, relational resources include discussion of advisors, friends, teachers, and mentors, that served as support or barriers to their participation in the field. When this connection happened in the narratives, many people discussed classes and programs (material resources) that led them to advisors, mentors, and friends (relational resources) who played critical roles in their participation in physics. We also see relational resources connect at least once with all the other CPI constructs except competence/performance, in instances where relationships with people have provided access to the other constructs. This could suggest that relationships have a similar impact as material resources for our participants. These people provided support by helping the subjects learn content, learn about and gain interest in the field, as well as deal with their personal struggles throughout their pursuit of the field.

Similarly, there were six times in our sample where positive material resources co-occurred with positive competence/performance, where things like classes and programs lead participants to discuss performing well and understanding physics content. The material resource connections with relational resources and competence/ performance were often unidirectional, with material resources often leading people to positive relationships and positive competence/performance. However, when material resources connected with interest, which happened less frequently, the directionality was inverted and positive content interest lead to positive material resources. We see an example of interest leading to a material resource in the second connection listed in Table I, where Oliver talks about the physics curriculum at his school being what attracted him to the department. Many participants discussed the ways that outreach programs and teaching acted as material resources that positively impacted their interest and participation in physics. This aligns with literature that reports the pattern of students of color in STEM wanting to give back to their communities [27]. In addition, 2 of the 5 times that internal positioning ideational resources were coded with positive material resources participants discussed the impacts of outreach. The other times material resources and interest connected was when participants discussed career goals, feelings of affirmation from participating in research and getting a sense purpose outside of just doing physics from performing spoken word.

\section{B. Internal positioning ideational resources and recognition}

In our prior work [26], we discussed the strong link between internal positioning ideational resources and recognition. In this data set, we found that three of the four recognition subcodes were coded in the same segments as internal positioning ideational resources: positive internal recognition, negative internal recognition, and negative external recognition. Internal codes connecting with other internal codes often could be a byproduct of the operationalization of the CPI. We see how the operationalized definitions of these codes can link them: recognition as a physicist from one's self (internal recognition) can influence how a person sees their positioning in the field (internal positioning). In Table I we see that positive internal recognition has the most co-occurrences with internal positioning ideational resources (10 co-occurrences). This connection occurs for most of the participants and is in a part of the narrative where a participant is stating that they identify as a physicist. Negative recognition had 8 cooccurrences with internal positioning ideational resources. This connection shows up in fewer interviews, but when it occurs, it is in a part of the narrative where participants are discussing when they do not completely feel like a physicist. In these cases, participants discuss things like the following: feeling like they do not fit the "stereotypical" ideas of a physicist, not fitting in classes, not having enough schooling in physics, or not attending prestigious enough schools to be considered a "real" physicist.

In our sample, when external recognition connects with internal positioning ideational resources the recognition is negative. From Table I, we see that there are 5 times that negative external recognition co-occurs with internal positioning ideational resources. When this connection happens in the narrative, participants discuss things like not feeling they belong in physics culture, assumptions that people make about them being a physicist, or their value as a physicist based on their appearance. We see this in the example from Cara in Table I, where she describes the tendency of others to not consider her a physicist because her Ph.D. is in Education, even though she has two physics degrees. We also see that negative external recognition connection occurred in 2 interviews from our sample, Cara and Erica, and 5 times total. In Cara's case, these were three separate times she received negative recognition from either a person more senior in the field (two professors and a higher-ranking colleague). In Erica's case, these were specifically related to negative recognition she received from family, who ostracized her because of her pursuit of higher education.

\section{DISCUSSION: BLACK PHYSICS IDENTITY}

\section{A. Revisiting research questions}

RQ 1: What CPI constructs are important for supporting Black physics identity?

From the data presented in the last section, we see that ideational resources and material resources were influential factors in the narratives of the physicists in our sample. In our sample, participants simultaneously do and do not see themselves as physicists. This mindset is informed by a disconnect between the personal goals and interests of our participants and what they perceive is valued in the physics field. Because ideational resources were the most coded of all the CPI constructs, we stress the importance of 
communicating ideologies to young Black physics students about what it means to position themselves as a Black physicist.

We propose two areas of action from these findings. First, we as physicists need to interrogate the cultural values of the field and find mechanisms to communicate (through ideational and material resources) that people with values outside of the traditional norms [8] are valued participants of the physics discipline. Second, we must regularly communicate with each other about the ways that real-world structures (material resources) and systems of oppression (ideational resources) operate in our discipline, while also making the resources that are available for Black physics professionals and students to persist despite them. The physics community needs a collective understanding that the experiences of Black physicists often includes unique challenges, but also includes common ideational and material barriers. This understanding is a baseline for changing the structures and systems that continue to exclude and push out Black members of the physics community. Simultaneously, this collective understanding might help equip students with the agency and autonomy needed to advocate for themselves and look for the resources they need while pursuing physics degrees.

Similar to prior studies, we see recognition as an important factor in the narratives of our participants. Our findings in this work suggest that recognition can impact the ways that our participants position themselves as physicists. We find that more junior participants discuss internal recognition more so than external ideas, and we find the opposite to be the case for more senior participants. However, for the women in our sample, the external recognition they discuss is mostly negative, where the men discuss mostly positive external recognition. These findings indicate several things. First, it is important for younger participants to recognize themselves as individuals capable of pursuing and succeeding in physics. We also see that more recognition is not always positive, and for women in our sample, being in the field longer meant enduring more negative recognition from others. For researchers, we stress the importance of future work to examine data along lines of intersecting identities like gender and sexuality. These intersecting identities elucidate important nuances in experience, as each Black physicist experiences oppression in unique ways. In future work, we plan to do more intersectional studies to understand this in more detail.

RQ 2: What are ways in which connections between CPI constructs provide support for Black physics identity?

For our sample, positive material resources connect most often and are often connected to other positive constructs. Because our findings show that ideational resources are most influential to our sample, we also look at the connections of other constructs to ideational resources to inform our analysis. When positive material resources connected with ideational resources, it was with the internal positioning subcode. The material resources discussed when this connection happened in our participants' narratives were almost always about outreach, research, and performing arts hobbies (e.g., spoken word). While participation in things like outreach and the arts were meaningful to our participants, we recognize that these material resources are less valued in the physics field overall. This points to the possibility that more valued material resources, like passing classes, while seen as important to the field, might not contribute largely to some Black physicists' identity.

In addition, we see a common connection between internal positioning ideational resources and recognition, both positive and negative. When positioning is connected with positive recognition, participants state that they identify as a physicist, and some mention how things like material resources (e.g., degrees) validate them as a physicist. However, when positioning is connected with negative recognition, participants discuss reasons why they do not fully identify as a physicist and feel welcomed in the physics field. Amongst the reasons why participants feel this way are explanations of ways that they do not align with the norms, values, and practices of physics culture. We see these connections from our findings as an indication that the material resources that were available to and taken up by our participants did not always positively influence these physicists' ideas of their position in physics. While positive material resources encouraged their participation in the field, many of them still see their participation either as something separate from their personal goals and interests or as a stepping stone to reaching non-traditional physics goals (i.e., teaching, inspiring, and mentoring other Black physics students).

\section{B. Implications: Physics culture}

A big lesson learned from this study is that not all barriers that Black physicists face while pursuing the field are material. Many of them are ideational and stem from implicit or explicit messages that are being received by Black folks about the norms, values, and practices of the physics discipline. These features are part of what makes up physics culture, and highly influence how Black students position themselves as members of the community (or not). These ideational resources are influenced by the racialized experiences Black physicists have to navigate on a daily basis, and from our findings, we know they can be directly impacted by material resources and internal and external recognition. In addition, the physicists in this study coped with their struggles by using nontraditional material resources like performance art as well as outreach. Many also discussed being motivated by interests other than physics research, like being interested in teaching over research.

In order to cultivate more Black physicists, the culture of physics is in need of an ideological shift towards values, practices, and norms that better support Black physics 
identity. The last two sections presented broad community actions to move towards this ideational shift. Here, we use the data in this study to suggest three ways we can do this through material and ideational resources. First, we must interrogate the material resources that we provide students from marginalized populations (i.e., bridge programs, research experiences, mentoring communities). How are these resources providing students with the positive recognition needed for them to position themselves as valid and legitimate members of the physics community while valuing their personal goals, interests, and reasons for being a member? This approach is particularly important for young physicists, as the young participants in this study got most of their recognition from themselves, and for women, who reported receiving more negative recognition at more senior positions. How do we shift the incentives and definitions of success in physics so that these programs are valued by the broader physics community? We suggest two approaches for answering these questions. First, providing awards and recognition for student, faculty, and staff participation in outreach and diversity-oriented trainings, incorporating the required norms of special programs for marginalized students into the requirements and norms of the entire student population of the department. Instead of requiring more from students involved in special bridge programs and pipeline programs, instilling these requirements department wide can even out the expectations for all students. In addition, coaching faculty in supporting students' self-defined ideas of success and career trajectory can be an important mentoring feature for the success of Black students.

Second, we must make a significant and intentional effort to breakvdown the harmful ideologies inherent in physics culture. A leading ideology that we see as harmful to physics culture is the idea that physics sits independently from our broader culture, and that it is not influenced by the systems of oppression and marginalization inherent in society (i.e., sexism, racism, homophobia, transphobia, and ableism). The spreading of this ideology is harmful to Black physicists, who know from personal experience that the racism they encounter daily does not disappear in physics spaces. It is, in fact, amplified at times, because of the collective denial perpetuated by the community's belief system that physics is without culture. When one experiences discrimination, they are often questioning if it is truly that, and when those experiences are denied by the community, the fault falls back on the person being victimized. At the community level, this denial can exacerbate the broader societal systems' marginalization and oppression by faulting the very people who are victimized. Addressing and acknowledging the culture of physics can happen through providing and requiring regular educational experiences like workshops or classes for faculty, staff, and administrators in physics departments and organizations. There exist resources for antioppression training, including ones that are physics specific [28], but many require time, energy, and money to implement. While many departments may claim a shortage of resources here, we suggest that such training be reframed through the job requirements for faculty and staff. In this way, elements of diversity and inclusion training can be incorporated into the educational and professional development experiences for all members of physics departments on an ongoing basis.

Third, we must be explicit about and value the many pathways and applications one can pursue and utilize with a degree in physics. The traditional methods of doing research in a lab or in theory are not always what bring Black folks into physics, and are often not what keeps them in the discipline. Further exploring new ways that physics can be learned, taught, and applied in the arts could create ideational resources about physics that welcomes alternative modes of participation. This approach can happen through language in department brochures and recruitment efforts, by including representation of nontraditional physicists and careers. Additionally, incorporating information about career paths into introductory and advanced physics courses could help normalize these options for students.

\section{CONCLUSION}

The ability to identify as a physicist is molded by one's experiences in relation to and within the field. Because Black physicists have racialized experiences in the field, we form unique identities as physicists and have different levels and forms of access to resources that are important for happy and healthy participation in the field. We know there are specific structural and systemic barriers that disproportionately affect students from marginalized groups. Like the lead author's own experience, material resources such as the physics GRE have been proven to be an example [29,30]. Structures like pipeline or bridge programs have served as material resources that look to address barriers like the GRE, but even these programs can reach only a fraction of marginalized students. The data presented here show how important ideational resources are in the experiences of the Black physicists in our sample. We argue for the need to complement efforts that focus on supplementing material resources with efforts that cultivate ideational resources that support Black physicists in receiving positive recognition and encourage them to position themselves as a physicist.

\section{ACKNOWLEDGMENTS}

We would first like to acknowledge and thank our study participants, who have shared their time, energy, and stories to contribute to this work. Additionally, this paper is based upon work supported by the NSF through a Graduate Research Fellowship, and Grants No. AISL 1713060 and No. DMR 1548924. Any opinions, findings, and conclusions or recommendations expressed in this material are those of the author(s) and do not necessarily reflect the views of the National Science Foundation. 


\section{APPENDIX A: CRITICAL PHYSICS IDENTITY CONSTRUCT DEFINITIONS}

The Critical Physics Identity (CPI) Framework was developed in prior work [20] to analyze interviews of Black physicists. Table II below outlines the updated codes that align with the six constructs of the CPI framework: material resources, relational resources, ideational resources, interest, recognition, competence/performance.

TABLE II.

\begin{tabular}{|c|c|c|}
\hline CPI construct & Subcode 1 & Subcode 2 \\
\hline \multirow[t]{4}{*}{$\begin{array}{l}\text { Ideational Resource: Aspect of an idea } \\
\text { that impacts one's connection to } \\
\text { physics }\end{array}$} & $\begin{array}{l}\text { Positioning in physics: ideas about one's } \\
\text { place in physics or how one negotiates } \\
\text { their position within the physics } \\
\text { community }\end{array}$ & $\begin{array}{l}\text { Internal: Your ideas (what you think) } \\
\text { External: Other's ideas (What other's } \\
\text { think) }\end{array}$ \\
\hline & $\begin{array}{l}\text { What is valued in physics: ideas about } \\
\text { what physicists care about and value } \\
\text { within the community }\end{array}$ & $\begin{array}{l}\text { Internal: Your ideas (what you think) } \\
\text { External: Other's ideas (What other's } \\
\text { think) }\end{array}$ \\
\hline & $\begin{array}{l}\text { Perceptions of physicists: cultural } \\
\text { perceptions about who or what } \\
\text { physicists are from those within and } \\
\text { outside of the field }\end{array}$ & $\begin{array}{l}\text { Internal: Your ideas (what you think) } \\
\text { External: Other's ideas (What other's } \\
\text { think) }\end{array}$ \\
\hline & $\begin{array}{l}\text { Personal characteristics: ideas about who } \\
\text { you are as a person (your struggles, } \\
\text { strengths and weaknesses, personality } \\
\text { traits, etc.) }\end{array}$ & $\begin{array}{l}\text { Internal: Your ideas (what you think) } \\
\text { External: Other's ideas (What other's } \\
\text { think) }\end{array}$ \\
\hline \multirow{3}{*}{$\begin{array}{l}\text { Material Resource: material things that } \\
\text { can provide access to other identity } \\
\text { constructs. (i.e., programs, } \\
\text { communities, organizations, funding) }\end{array}$} & Positive: provides access to the field & $\mathrm{N} / \mathrm{A}$ \\
\hline & Negative: prevents access to the field & $\mathrm{N} / \mathrm{A}$ \\
\hline & $\begin{array}{l}\text { Neither: it is not clear whether it } \\
\text { provided or prevented access to the } \\
\text { field }\end{array}$ & $\mathrm{N} / \mathrm{A}$ \\
\hline \multirow{3}{*}{$\begin{array}{l}\text { Relational resource: the way in which an } \\
\text { aspect of a relationship with others in } \\
\text { the context can impact one's } \\
\text { connection to physics }\end{array}$} & $\begin{array}{l}\text { Positive: when an interviewee mentions } \\
\text { a unique aspect of a relationship that } \\
\text { encouraged them to participate }\end{array}$ & $\mathrm{N} / \mathrm{A}$ \\
\hline & $\begin{array}{l}\text { Negative: when an interviewee mentions } \\
\text { a unique aspect of a relationship that } \\
\text { discouraged them to participate }\end{array}$ & $\mathrm{N} / \mathrm{A}$ \\
\hline & $\begin{array}{l}\text { Neither: when an interviewee mentions a } \\
\text { unique aspect of a relationship that } \\
\text { was impactful, but they do not specify } \\
\text { whether it encouraged or discouraged } \\
\text { their participation. }\end{array}$ & $\mathrm{N} / \mathrm{A}$ \\
\hline \multirow{5}{*}{$\begin{array}{l}\text { Recognition: being recognized } \\
\text { (or not) as a physicist or } \\
\text { physics person }\end{array}$} & $\begin{array}{l}\text { Internal: when an interviewee mentions } \\
\text { getting recognition from themselves }\end{array}$ & $\begin{array}{l}\text { Positive: Recognition that encourages } \\
\text { participation in physics }\end{array}$ \\
\hline & as being a physicist & $\begin{array}{l}\text { Negative: Recognition that discourages } \\
\text { participation in physics } \\
\text { Neither: Recognition that is not clear } \\
\text { whether it encouraged or discouraged } \\
\text { their participation. }\end{array}$ \\
\hline & $\begin{array}{l}\text { External: when an interviewee mentions } \\
\text { getting recognition from others as }\end{array}$ & $\begin{array}{l}\text { Positive: Recognition that encourages } \\
\text { participation in physics }\end{array}$ \\
\hline & physicist & $\begin{array}{l}\text { Negative: Recognition that discourages } \\
\text { participation in physics }\end{array}$ \\
\hline & & $\begin{array}{l}\text { Neither: Recognition that is not clear } \\
\text { whether it encouraged or discouraged } \\
\text { their participation. }\end{array}$ \\
\hline
\end{tabular}


TABLE II. (Continued)

\begin{tabular}{|c|c|c|}
\hline CPI construct & Subcode 1 & Subcode 2 \\
\hline \multirow[t]{6}{*}{$\begin{array}{l}\text { Interest: Interest in the physics } \\
\text { field }\end{array}$} & Content: Interest in physics content & $\begin{array}{l}\text { Positive: Encouraging participation in } \\
\text { physics }\end{array}$ \\
\hline & & $\begin{array}{l}\text { Negative: Discouraging participation } \\
\text { in physics }\end{array}$ \\
\hline & $\begin{array}{l}\text { Noncontent: interest in physics for } \\
\text { reasons other than content }\end{array}$ & $\begin{array}{l}\text { Positive: Encouraging participation } \\
\text { in physics }\end{array}$ \\
\hline & & $\begin{array}{l}\text { Negative: Discouraging participation } \\
\text { in physics }\end{array}$ \\
\hline & $\begin{array}{l}\text { Teaching and Outreach: interest in } \\
\text { education, teaching, and outreach or }\end{array}$ & $\begin{array}{l}\text { Positive: Encouraging participation } \\
\text { in physics }\end{array}$ \\
\hline & giving back & $\begin{array}{l}\text { Negative: Discouraging participation } \\
\text { in physics }\end{array}$ \\
\hline \multirow{3}{*}{$\begin{array}{l}\text { Competence/performance: Belief } \\
\text { in ability to understand } \\
\text { and perform required } \\
\text { tasks }\end{array}$} & $\begin{array}{l}\text { Positive: when an interviewee mentions } \\
\text { good performance and/or competence } \\
\text { in physics content }\end{array}$ & $\mathrm{N} / \mathrm{A}$ \\
\hline & $\begin{array}{l}\text { Negative: when an interviewee mentions } \\
\text { poor performance and/or competence } \\
\text { in physics content }\end{array}$ & $\mathrm{N} / \mathrm{A}$ \\
\hline & $\begin{array}{l}\text { Neither: when an interviewee mentions } \\
\text { performance and/or competence in } \\
\text { physics content but is not clear } \\
\text { whether it is positive or negative. }\end{array}$ & $\mathrm{N} / \mathrm{A}$ \\
\hline
\end{tabular}

\section{APPENDIX B: INTERVIEW VALIDATION TABLE}

The interviews conducted for this work varied in time, but this did not impact the total number of codes that were found in each interview. We show this in Table III below which includes the duration of each interview from the participants in our sample, along with the total number of codes from the CPI framework that were found in each interview.

TABLE III. The code counts, duration, and code count per minute ratio for each interview in the 11 person sample.

\begin{tabular}{lccc}
\hline \hline Subject & Code counts & Duration (mins) & Counts/min \\
\hline Cara & 105 & 76.5 & 1.37 \\
Erica & 103 & 58.51 & 1.76 \\
Ronnie & 67 & 64.14 & 1.04 \\
Tammy & 51 & 46.07 & 1.11 \\
Alicia & 49 & 29.52 & 1.66 \\
Lisa & 45 & 36.01 & 1.25 \\
Andrew & 88 & 40.45 & 2.18 \\
Antwan & 78 & 49.07 & 1.59 \\
Owen & 81 & 57.48 & 1.41 \\
Oliver & 58 & 36.22 & 1.60 \\
Dylan & 90 & 45.4 & 1.98 \\
\hline \hline
\end{tabular}


[1] R. Czujko, R. Ivie, and J.H. Stith, Untapped Talent: The African American Presence in Physics and the Geosciences, AIP Report. Number R-444, Statistical Research Center of the American Institute of Physics (AIP, New York, 2008), http://eric.ed.gov/?id=ED503414.

[2] B. A. Brown, C. Mangram, K. Sun, K. Cross, and E. Raab, Representing racial identity: Identity, Race, the construction of the African American STEM students, Urban Educ. 52, 170 (2017).

[3] A. Byars-Winston, J. Rogers, J. Branchaw, C. Pribbenow, R. Hanke, and C. Pfund, New measures assessing predictors of academic persistence for historically underrepresented racial/ethnic undergraduates in science, $\mathrm{CBE}$ Life Sci. Educ. 15 (2016).

[4] A. Johnson, J. Brown, H. Carlone, and A. K. Cuevas, Authoring identity amidst the treacherous terrain of science: A multiracial feminist examination of the journeys of three women of color in science, J. Res. Sci. Teach. 48, 339 (2011).

[5] M. Ong, Body projects of young women of color in physics: Intersections of gender, race, and science, Social problems 52, 593 (2005).

[6] L. T. Ko, R. R. Kachchaf, A. K. Hodari, and M. Ong, Agency of women of color in physics and astronomy: Strategies for persistence and success, J. Women Minorities Sci. Engin. 20, 171 (2014).

[7] L. T. Ko, R. R. Kachchaf, M. Ong, and A. K. Hodari, Narratives of the double bind: Intersectionality in life stories of women of color in physics, astrophysics and astronomy, AIP Conf. Proc. 1513, 222 (2013).

[8] S. Traweek, Beamtimes and Lifetimes: The World of High Energy Physicists (Harvard University Press, Cambridge, MA, 1988).

[9] T. J. Atherton, R. S. Barthelemy, W. Deconinck, M. L. Falk, S. Garmon, E. Long, and K. Reeves, LGBT climate in physics: Building an inclusive community (American Physical Society, College Park, MD, 2016), pp. 118-126.

[10] K. Rosa and F. M. Mensah, Educational pathways of Black women physicists: Stories of experiencing and overcoming obstacles in life, Phys. Rev. Phys. Educ. Res. 12, 020113 (2016).

[11] E. O. McGee and L. Bentley, The troubled success of black women in STEM, Cognit. Instr. 35, 265 (2017).

[12] E. O. McGee, Devalued Black and Latino Racial Identities: A By-Product of STEM College Culture?, Am. Educ. Res. J. 53, 1626 (2016).

[13] D. Li and C. Koedel, Representation and salary gaps by race-ethnicity and gender at selective public universities, Educ. Res. 46, 343 (2017).

[14] S. Fries-Britt, T. Younger, and W. Hall, Underrepresented minorities in physics: How perceptions of race and campus climate affected student outcomes, Managing Diversity: (Re)visioning Equity on College Campuses, edited by T. Elon Dancy II (Peter Lang Publishing, New York, 2010), pp. 181-196.

[15] S. L. Fries-Britt and K. M. Holmes, Prepared and progressing: Black women in physics, Black Female Undergraduates on Campus: Successes and Challenges, edited by C. R. Chambers and R. V. Sharpe (Emerald Group Publishing Limited, United Kingdom, 2012), pp. 199-218.

[16] S. Fries-Britt, J. Johnson, and B. Burt, Black students in physics: The intersection of academic ability, race, gender, and class, Living at Intersections, edited by T. L. Strayhorn (Information Age Publishing, Inc., Charlotte, NC, 2013), pp. 21-39.

[17] E. Wenger, Communities of practice and social learning systems: the career of a concept, Social Learning Systems and Communities of Practice (Springer, New York, 2010), pp. 179-198.

[18] Z. Hazari, G. Sonnert, P. Sadler, and M. Shanahan, Connecting high school physics experiences, outcome expectations, physics identity, and physics career choice: A gender study, J. Res. Sci. Teach. 47, 978 (2010).

[19] E. D. Tate and M. C. Linn, How does identity shape the experiences of women of color engineering students?, J. Sci. Educ. Technol. 14, 483 (2005).

[20] S. Hyater-Adams, C. Fracchiolla, N. Finkelstein, and K. Hinko, Critical look at physics identity: An operationalized framework for examining race and physics identity, Phys. Rev. Phys. Educ. Res. 14, 010132 (2018).

[21] M. Esteban-Guitart and L. C. Moll, Funds of Identity: A new concept based on the Funds of Knowledge approach, Culture Psychol. 20, 31 (2014).

[22] N. Nasir, Racialized Identities: Race and Achievement among African American Youth (Stanford University Press, Stanford, California 2011).

[23] F. M. Connelly and D. J. Clandinin, Stories of experience and narrative inquiry, Educ. Res. 19, 2 (1990).

[24] F. Wilcoxon, S. K. Katti, and R. A. Wilcox, Critical values and probability levels for the Wilcoxon rank sum test and the Wilcoxon signed rank test, Selected Tables Math. Stat. 1, 171 (1970).

[25] J. Cohen, Statistical Power Analysis for the Behavioral Sciences. Retrieved from (2013) https://books.google.com/ books?id=2v9zDAsLvA0C.

[26] S. Hyater-Adams, C. Fracchiolla, K. Hinko, and N. Finkelstein, Understanding connections between physics and racial identities through recognition and relational resources, in Proceedings of the 2016 Physics Education Research Conference, Sacramento, CA, edited by D. L. Jones, L. Ding, and A. Traxler (AIP, New York, 2016), pp. $164-167$.

[27] C. Y. Umanzor, Investigating the experiences of graduate women of color in biology, Ph.D. thesis, San Francisco State University 2011.

[28] A. Daane, S. R. Decker, and V. Sawtelle, Teaching about racial issues in introductory physics courses, Phys. Teach. 55, 328 (2017).

[29] C. Miller and K. Stassun, A test that fails, Nature (London) 510, 303 (2014).

[30] G. Potvin, D. Chari, and T. Hodapp, Investigating approaches to diversity in a national survey of physics doctoral degree programs: The graduate admissions landscape, Phys. Rev. Phys. Educ. Res. 13, 020142 (2017). 